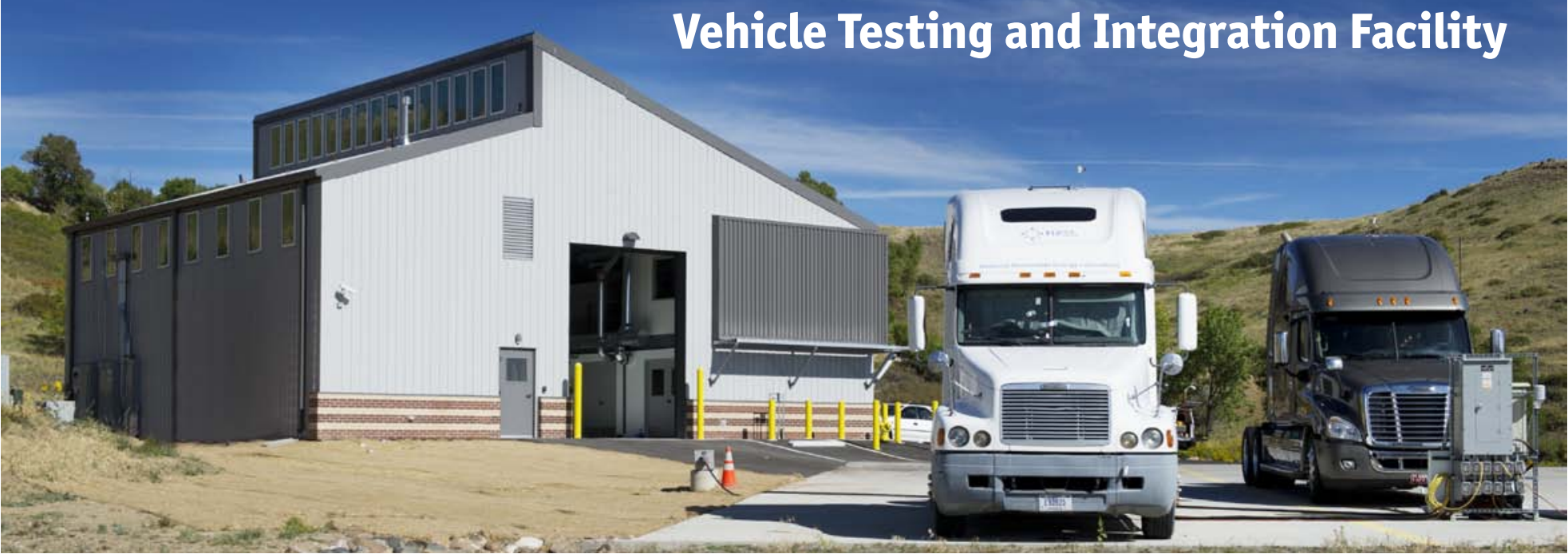

Photo by Dennis Schroeder, NREL/PIX 19932

\title{
Integrated PEV Charging Solutions and Reduced Energy for Occupant Comfort
}

Plug-in electric vehicles (PEVs) offer the opportunity to shift transportation energy demands from petroleum to electricity, but broad adoption will require integration with other systems. While automotive experts work to reduce the cost of PEVs, fossilfueled cars and trucks continue to burn hundreds of billions of gallons of petroleum each year-not only to get from point $A$ to point $B$, but also to keep passengers comfortable with air conditioning and heat.

At the National Renewable Energy Laboratory (NREL), three installations form a research laboratory known as the Vehicle Testing and Integration Facility (VTIF). At the VTIF, engineers are developing strategies to address two separate but equally crucial areas of research: meeting the demands of electric vehicle-grid integration and minimizing fuel consumption related to vehicle climate control.

Part of NREL's Center for Transportation Technologies and Systems (CTTS), the VTIF is dedicated to renewable and energyefficient solutions. This facility showcases technology and systems designed to increase the viability of sustainably powered vehicles. NREL researchers instrument every class of on-road vehicle, conduct hardware and software validation for electric vehicle (EV) components and accessories, and develop analysis tools and technology for the Department of Energy, other government agencies and industry partners.

Research conducted at the VTIF examines the interaction of building energy systems, utility grids, renewable energy sources and PEVs, integrating energy management solutions, and maximizing potential greenhouse gas (GHG) reduction, while smoothing the transition and reducing costs for EV owners. NREL's collaboration with automakers, charging station manufacturers, utilities and fleet operators to assess technologies using VTIF resources is designed to enable PEV communication with the smart grid and create opportunities for vehicles to play an active role in building and grid management. Ultimately, this creates value for the vehicle owner and will help renewables be deployed faster and more economically, making the U.S. transportation sector more flexible and sustainable.

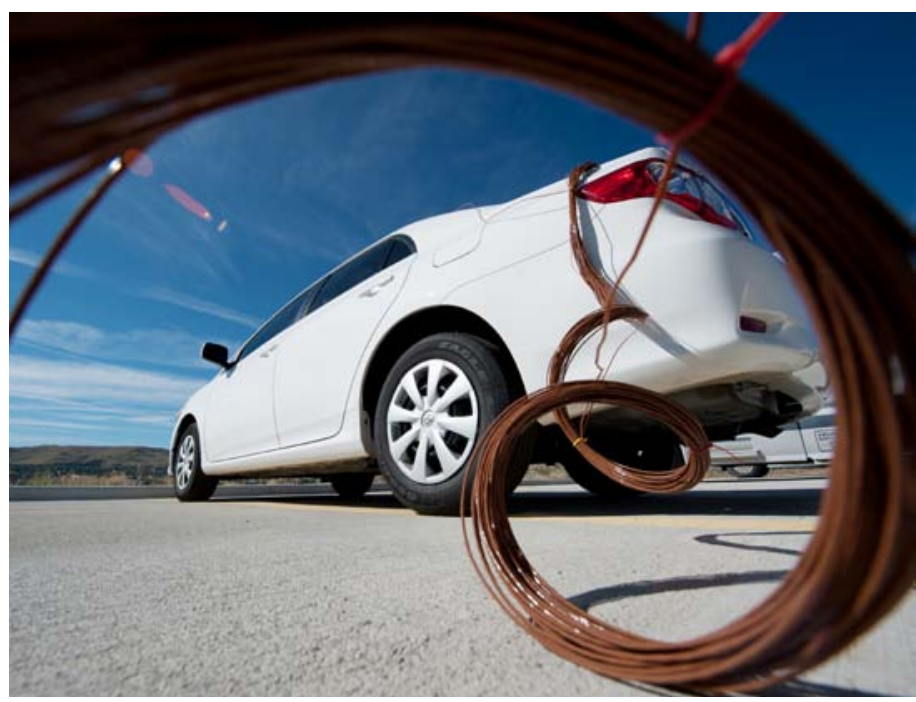

Car undergoing climate control analysis at the VTIF. Photo by Dennis Schroeder, NREL/PIX 19931 


\section{Vehicle Instrumentation}

\section{Primary Use}

The VTIF accommodates a wide range of vehicles for modification and testing. Society of Automotive Engineers, Institute of Electrical and Electronics Engineers, and Institute of Energy Conversion standards related to vehicle charging and communication technology are advanced through vehicle modification projects. A machine shop and circuits lab make it possible to complete vehicle modifications and instrumentation in-house.

\section{Facilities \& Features}

- Four bays (three passenger, one heavy vehicle) for vehicle modifications, instrumentation set-up and experiments

- Room to accommodate high-clearance vehicles

- One 50kW DC fast PEV charger (CHAdeMO)

- Three outdoor AC Level 2 electric vehicle supply equipment (EVSE) units

- One wireless vehicle charging station

- Electronics fabrication, machine and welding shops

- $A C$ and DC bus lines to interconnect solar energy sources, vehicles, energy storage, smart grid controls and emulated renewables

- 24 locking high-power outlets to accommodate future EV charging components or renewable energy systems

- Approximately 2,800 sq. ft.

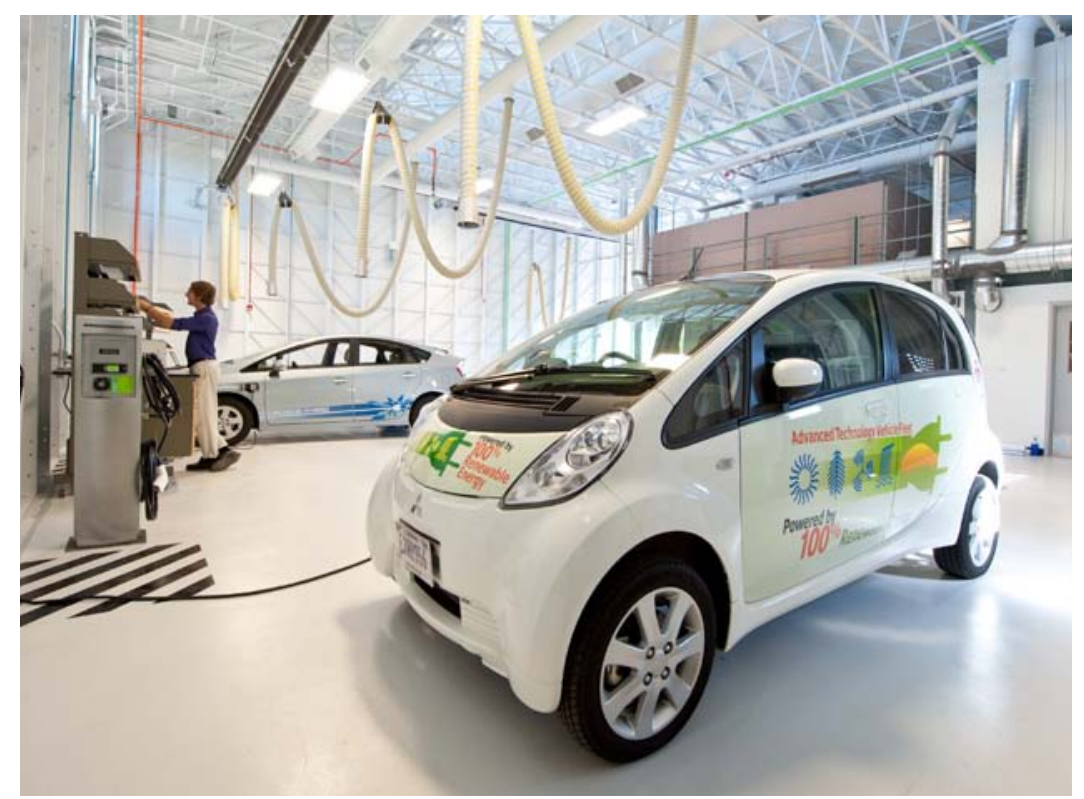

PEV charging in the VTIF. Photo by Dennis Schroeder, NREL/PIX 19758

\section{LEED-Gold Certified}

The VTIF has been designated a Leadership in Energy and Environmental Design (LEED) facility by the U.S. Green Building Council. Gold certification means that this is one of the most environmentally friendly structures in the country. Its green features include:

- Water-efficient fixtures and landscaping

- Energy efficient systems

- Recycled and regionally sourced construction materials

- Low-emitting paints, sealants and flooring

- Daylighting and lighting controls

\section{EV Infrastructure Highlights}

VTIF EV infrastructure accommodates the testing of EVSE, vehicle and grid interaction. This flexible system provides full monitoring and control at each grid access point. Charging points can range from $3 \mathrm{~kW}$ to $150 \mathrm{~kW}$ using $\mathrm{AC}$, DC and wireless power transfer technology.

- Bi-directional energy management of PEVs

- Public fast charging in 15 to 30 minutes

- Simultaneous charging of multiple vehicles

- Wireless drive-on vehicle charging

- Charging control via ethernet, WiFi, WiMax, ZigBee, USB, satellite, RFID, ModBus, smartphone and serial connections

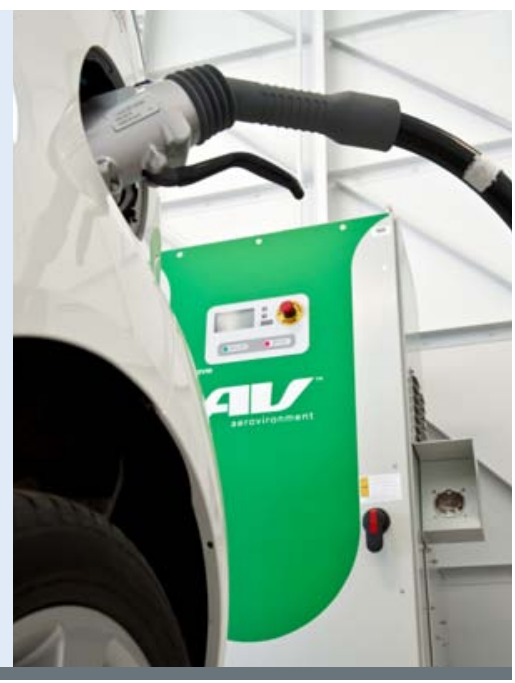




\section{Vehicle Thermal Testing}

\section{Primary Use}

The VTIF site provides a south-facing secure test pad for conducting multiple concurrent light and heavy vehicle thermal soak testing and stationary HVAC load testing.

\section{Facilities \& Features}

Designed to support vehicle thermal efficiency research in areas including:

- Light- and heavy-duty vehicle climate control load reduction

- Minimization of the impact of climate control on EV range

- Minimization of heavy-duty vehicle fuel consumed by engine idling for climate control

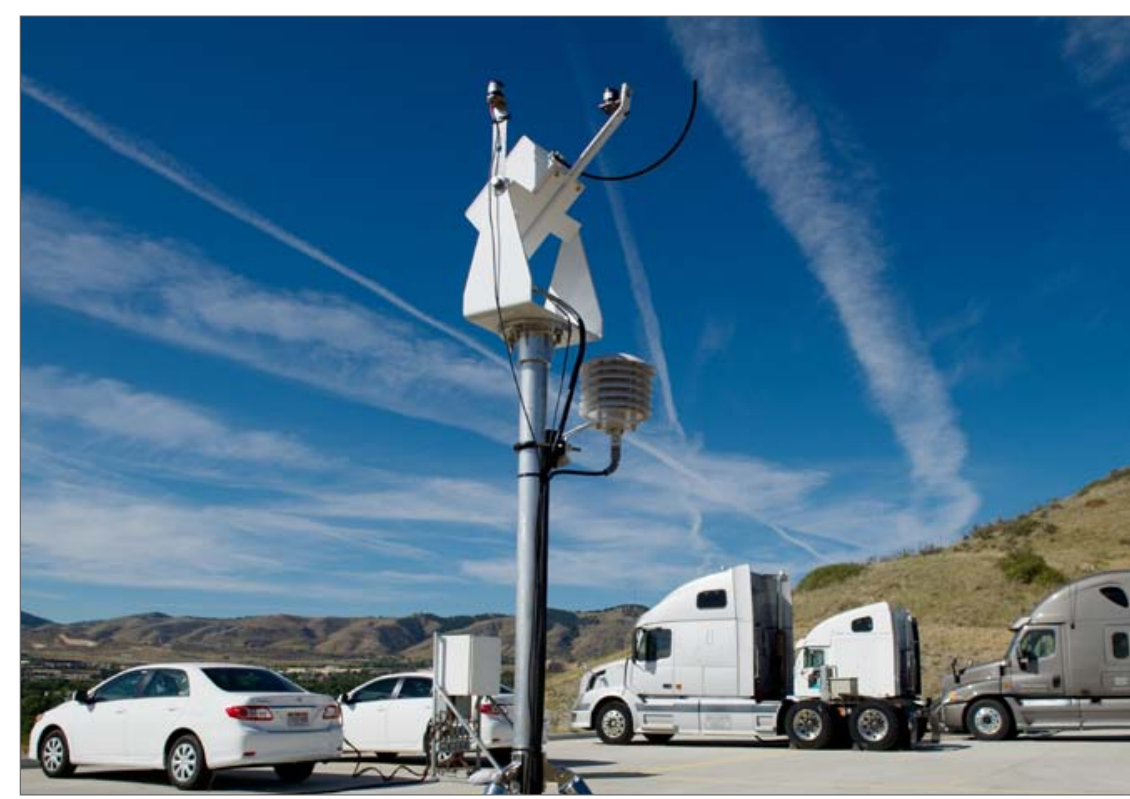

Weather station and vehicles on the VTIF test pad. Photo by Dennis Schroeder, NREL/PIX 19930

- Approximately 10,000 sq. ft., enough space to accommodate simultaneous thermal testing of up to six Class 8 trucks

- South-facing vehicle orientation with minimal shading from surrounding environment for maximum solar exposure

- High-fidelity weather station in close proximity to the test pad, providing continuous data stream on temperature, humidity, wind speed and solar energy intensity

- Convenient power pedestals supplying ample energy for computers, data acquisition systems and grid-powered climate control systems

\section{Impact of HVAC Systems}

- Each year, air conditioning light-duty vehicles in the U.S. consumes 7 billion gallons of gasoline

- EV range is reduced $30 \%-45 \%$ due to heating and air conditioning

- Overnight idling of long-haul trucks for driver comfort burns 838 billion gallons of diesel annually

\section{VTIF Testing, Analysis and Demonstration Capabilities}

\section{Vehicle Energy Management with Smart Grid}

- Optimize vehicle energy flow with residential grids and distributed renewables

- Manage energy demand in military and commercial settings to reduce costs and increase robustness

- Create information flow requirements and support standards development efforts

- Coordinate multiple PEVs and EVSEs with smart grid

- Simulate smart grid, energy storage, renewables and PEV system scenarios

\section{PEV Charge Integration with Renewables Research}

- Demonstrate grid-connected vehicle load-supply coordination using renewable and conventional energy sources

\section{Off-Board Bidirectional Vehicle Charger Testing \& Demonstration}

- Identify and quantify potential efficiency, cost and integration benefits of DC off-board PEV charging

\section{EVSE Testing \& Verification}

- Assess response of PEVs and EVSEs to grid communications

\section{Vehicle Thermal Management}

- Reduce EV climate control loads

- Decrease heavy-duty vehicle idle requirements

- Assess the impact of insulation, reflective materials and other vehicle modifications on thermal loads

- Predict the effect of thermal loads on fleet fuel consumption and related expenses 


\section{Grid and Vehicle Systems Integration}

\section{Primary Use}

This experimental system enables hardware and hardware-in-theloop simulation of command, communication and control systems for vehicle-to-grid integration, while maximizing the benefits of energy provided by renewable resources (e.g. , wind and solar).

\section{Facilities \& Features}

- Purpose-built multi-bus-configurable distribution grid to support advanced energy management concepts

- PEV "fueling" station with four parking spaces
- Solar tree with tracking system ( 20kW) —enough energy to deliver more than 300 electrified miles per day

- Ability to simulate wind turbines or loads from other sources

- Two DC fast chargers - up to $150 \mathrm{~kW}$

- EVSEs from multiple vendors, providing resources to investigate various methods of Level 2 charging

- Low-power DC charging facilities for integrating with building DC power networks

- Community energy storage system

- Smart grid interface monitor and controls

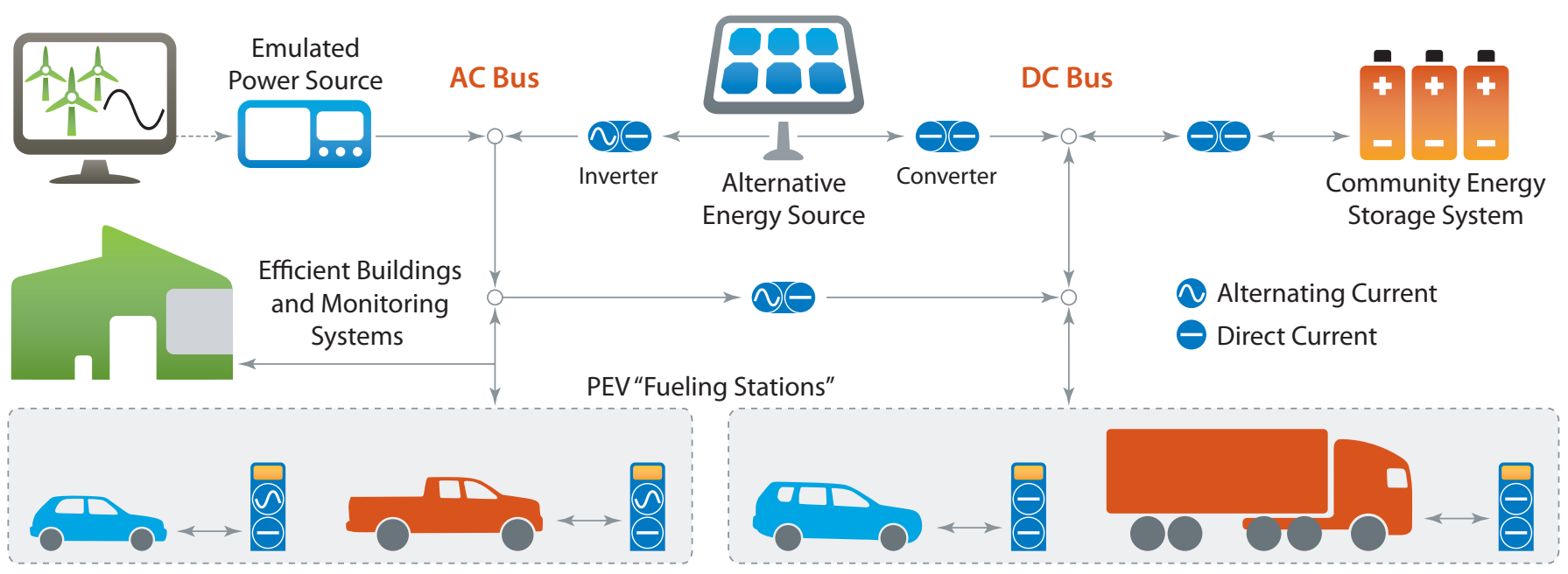

\section{About the Center for Transportation Technologies and Systems}

An arm of the only national laboratory dedicated to renewable energy and energy efficiency, NREL's Center for Transportation Technologies and Systems (CTTS) is at the forefront of R\&D for tomorrow's sustainable transportation solutions. The center's innovative and integrated whole-system approach helps government, industry and other research partners develop market-ready, high-performance, low-emission, fuelefficient vehicles, components and systems.

\section{About the CTTS Testing and Analysis Group}

The CTTS Testing and Analysis Group conducts in-use evaluations, complex computer analyses and simulations of advanced technology vehicles to help quantify the benefits of future transportation solutions; tests medium- and heavy-duty vehicles in a controlled laboratory environment; and investigates the issues associated with large-scale integration of plug-in vehicles and the utility grid.

For more information on the Vehicle Testing and Integration Facility (VTIF), contact Tony Markel: (303) 275-4478 or Tony.Markel@nrel.gov. 